\title{
A Novel Technique for the Treatment of a Case of Verneuil's Disease of Perineum and Axillary Regions
}

\author{
Etienne El-Helou, MD ${ }^{1}$ Alaa Kansoun, MD ${ }^{1} \quad$ Elissa Abi Fadel, MD ${ }^{2}$ Ali Nassif, MD ${ }^{1}$ \\ Houssam Bashir Mazraani, MD ${ }^{1}$ Georges Robert Neaime, MD ${ }^{1}$ Houssein Amin Kassem Moussa, MD ${ }^{10}$ \\ Georges Bassil, MD ${ }^{1}$ (i) Serge Ibrahim, MD ${ }^{1}$ Georges R. Assaf, MD ${ }^{3}$ Houssam Alam, MD 4
}

${ }^{1}$ Department of General Surgery, Lebanese University, Faculty of Medical Sciences, Beirut, Lebanon

2 Department of General Medicine, Lebanese University, Faculty of Medical Sciences, Beirut, Lebanon

${ }^{3}$ Department of Anesthesiology, Centre Hospitalier Universitaire Geitaoui, Lebanon

${ }^{4}$ Department of General Surgery, Centre Hospitalier Universitaire Geitaoui, Lebanon

\author{
Address for correspondence Etienne El-Helou, MD, Department of \\ General Surgery, Lebanese University, Faculty of Medical Sciences, \\ Beirut, Lebanon (e-mail: etienne-elhelou@hotmail.com).
}

Surg J (NY) 2021;7:e163-e167.

\begin{abstract}
Keywords

- Verneuil

- hidradenitis suppurativa

- Etanercept

- Integra

- synthetic

- case report

- novel technique

Hidradenitis suppurativa (HS) is a chronic inflammatory disease involving apocrine glands of the skin. It carries out an important burden on the daily life of the patient. Unfortunately, it presents a major concern for medical care management in the absence of clear guidelines for proper medical and surgical treatment. Hence, we report a case of concomitant axillary and perianal HS. We opted for surgical management using a novel technique, which proved efficacy for a year of follow-up recurrence free.
\end{abstract}

Hidradenitis suppurativa (HS), acne inversa, and Verneuil's disease are relatively the identical terminologies. ${ }^{1}$ It was described first in the 19th century by the French surgeon Aristide Auguste Stanislas Verneuil ${ }^{2}$ and since then numerous guidelines were reported worldwide concerning the management. However, they failed to prove efficacy. ${ }^{1}$ We report a case of a young male, with wide deep hidradenitis suppurativa of perineum and axillae, managed and followed out for 1 year, without recurrence.

\section{Case Description}

We report a case of a 30-year-old male with a history of hidradenitis suppurativa presented to our clinics with flare up of his disease with perineal and bilateral axillary ulcerative and purulent lesions. The history of his disease dates back to the age of 18 when he was treated medically with Etanercept and kept off symptoms for 10 years, after which he was treated surgically with repetitive incisions and debridement of recurrent small abscesses until this presentation.

On physical examination, the patient was febrile (temperature $=40.1^{\circ} \mathrm{C}$ ), tachycardic ( 110 beats per minute), normotensive (blood pressure $=120 / 80 \mathrm{mmHg}$ ), and he had normal oxygen saturation $\left(\mathrm{SpO}_{2}=97 \%\right)$. Multiple scares were noted over all his body, large bilateral axillary Hurley stage 2 abscesses, and perineal Hurley stage 3 lesions were noted. They were hot and tender to touch and with purulent secretions.

Moreover, the patient's laboratory studies revealed leukocytosis (white blood cell $=21,200 / \mu \mathrm{L})$ and CRP $(220 \mathrm{mg} / \mathrm{L}$ ). received

January 9, 2021

accepted

April 12, 2021
DOI https://doi.org/

$10.1055 / \mathrm{s}-0041-1731400$.

ISSN 2378-5128. (c) 2021. The Author(s).

This is an open access article published by Thieme under the terms of the Creative Commons Attribution License, permitting unrestricted use, distribution, and reproduction so long as the original work is properly cited. (https://creativecommons.org/licenses/by/4.0/)

Thieme Medical Publishers, Inc., 333 Seventh Avenue, 18th Floor, New York, NY 10001, USA 

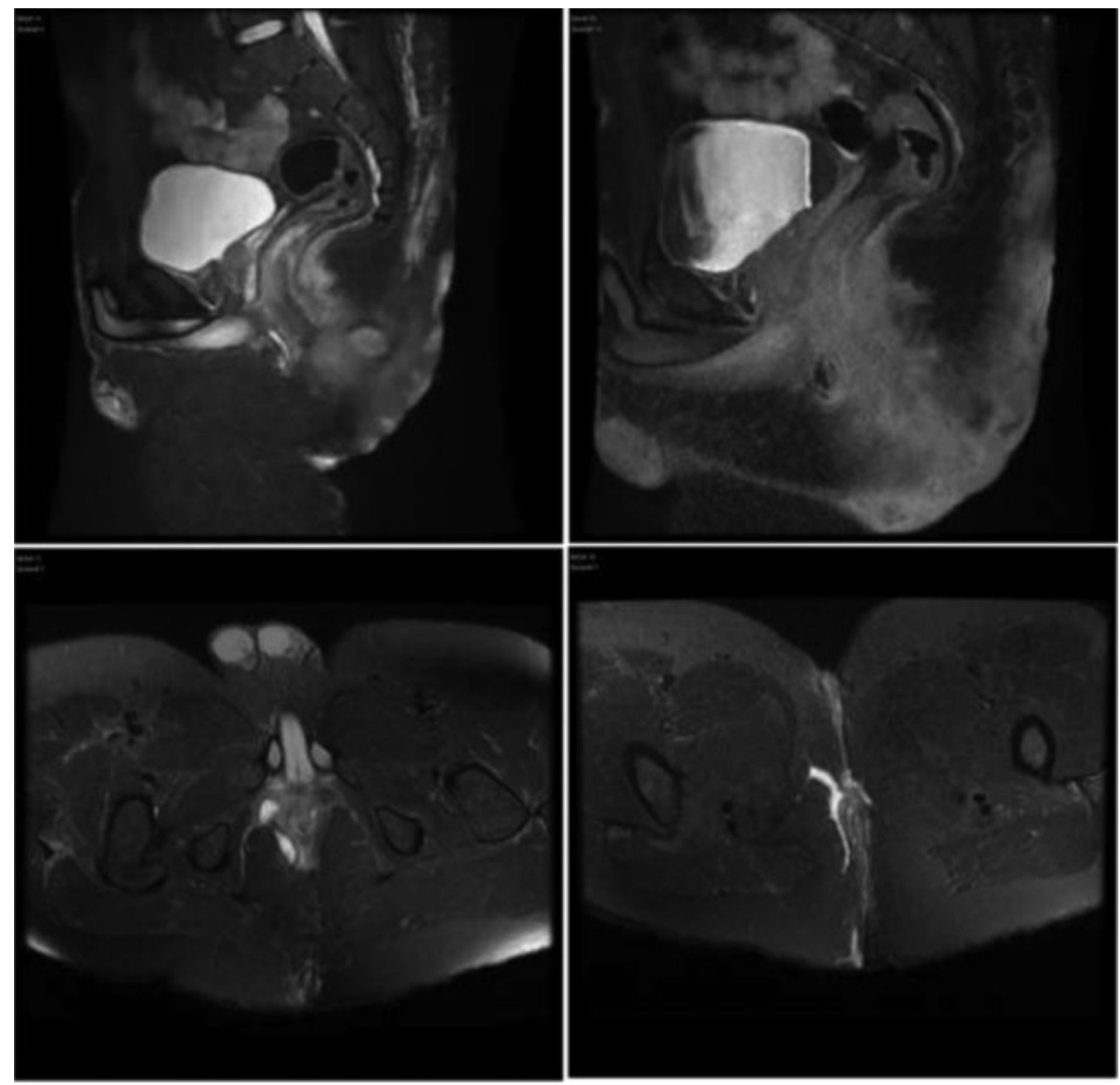

Fig. 1 Magnetic resonance imaging of pelvis showing multiple fistulous tract and abscesses.

Interestingly, Magnetic resonance imaging of the pelvis showed complex trans-sphincteric fistula at 12 o'clock with the presence of intersphincteric, anterior perirectal, and right ischial anal fossa abscesses as well as a fistulous tract within the right perineum, right buttocks, and extending from the perineum anteriorly to the intergluteal cleft posteriorly (-Fig. 1). Computed tomography of the chest showed cutaneous thickening associated with multiple air pockets bilaterally and almost symmetrically, with evidence of well circumscribed collections.

Broad spectrum antibiotherapy was started as well as multiple attempts of debridement, within few days interval, as a primary treatment of sepsis. Culture samples were in favor of carbapenem-resistant Enterobacteriaceae growth.

After a 6-week regimen of antibiotics, a radical excision of the lesions was done, keeping behind a wide open wound of perineum ( - Fig. 2 ) and bilateral wounds of axillae ( - Fig. 3 ). On day 2 , a protective side colostomy was done at the level of the descending colon to divert feces.

Negative pressure wound therapy (Genadyne's NPWT) was initiated over the three open wounds and continued for 2 months after surgery until the granulation tissue formation was noted (-Fig. 4).
The patient then benefited from intraoperative skin substitutes (Integra Dermal Regeneration template) placement over the open wounds and fixation with staples (-Figs. 5 and 6).

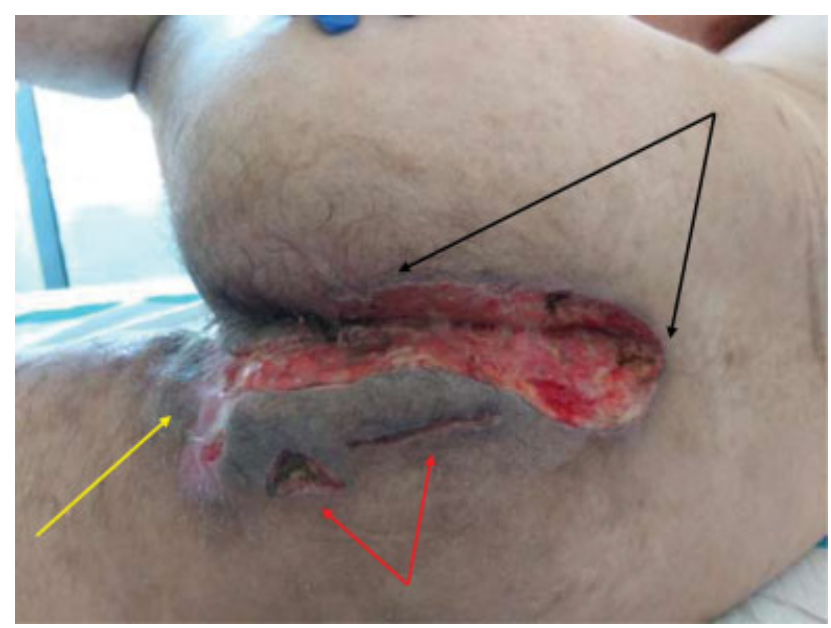

Fig. 2 Postdebridement of perineal abscesses (black arrows), after secondary healing of drained perianal lesion (yellow arrow), and multiple incision and drainage sites (red arrows). This photo is 1 day before surgical creation of colostomy. 


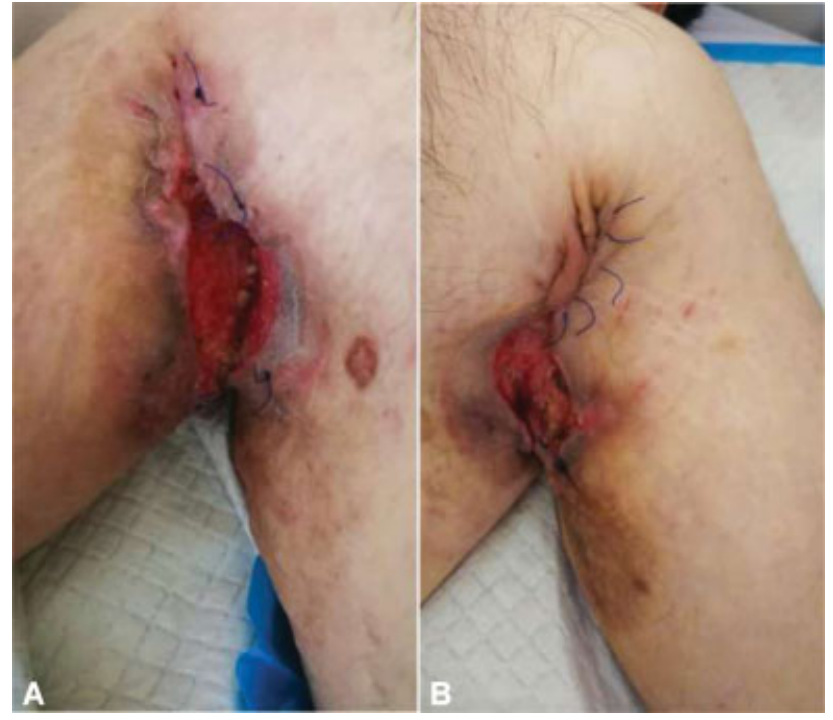

Fig. 3 Axillary lesions excision of abscesses: (A) right axillae and (B) left axillae. Primary suture of the superior part. The inferior part kept open for drainage.

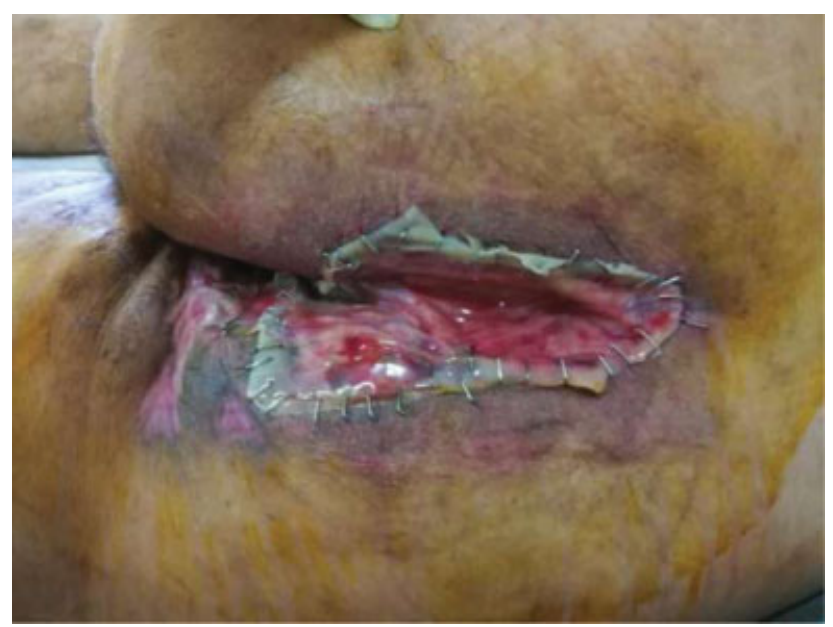

Fig. 5 Perineal integra dermal regeneration template TM insertion and fixation.

About 3 weeks postinsertion, the follow-up demonstrated good uptake of the graft without breakdown. Surgical staples were removed and patient was discharged home with instruction of wet to dry dressing with active Leptospermum honey (MEDIHONEY Gel) ( - Figs. 7 and 8 ).

Moreover, 1-year follow-up showed no recurrence and preservation of patient functionality ( - Fig. 9). Re-establishment of continuity of bowels done and patient discharged home without complications.

\section{Discussion}

HS is a chronic inflammatory disease of the skin, with a significant recurrence and burden on the affected persons, making them infirm and anxious. ${ }^{1}$ The nomenclature "hidradenitis suppurativa" is unsuitably applied, since the development of the disease involves the apocrine gland ${ }^{2}$ associated with systemic inflammation. ${ }^{2}$

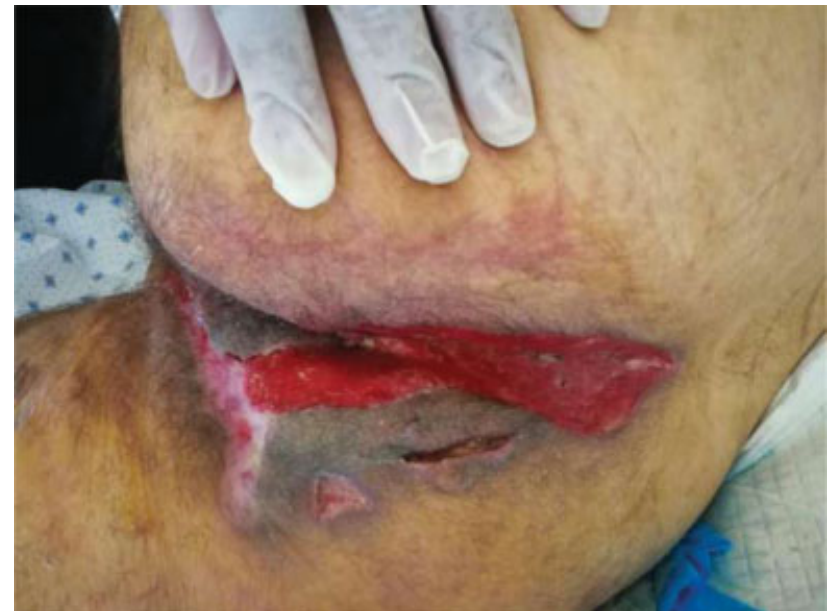

Fig. 4 Postperineal debridement and vacuum therapy for 2 months. Granulation tissue formation noted.

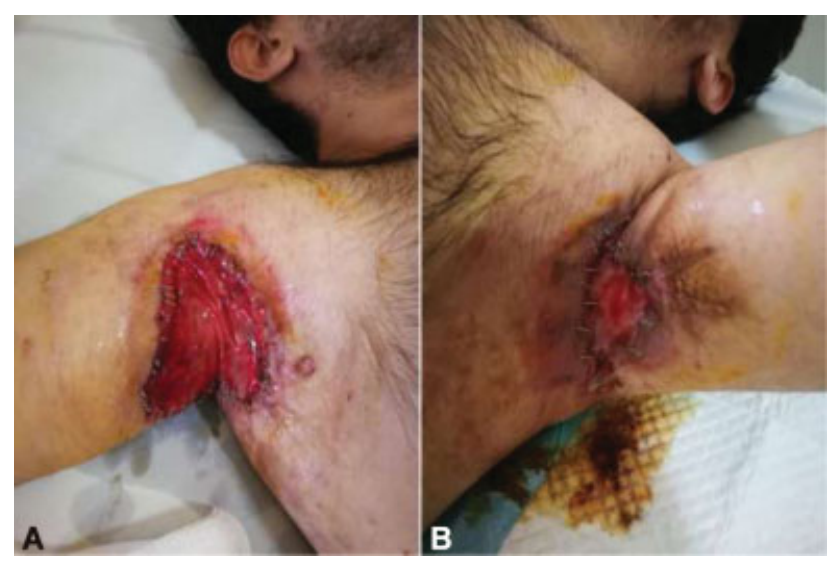

Fig. 6 Right (A) and left (B) axillary Integra Dermal Regeneration template TM insertion and fixation.

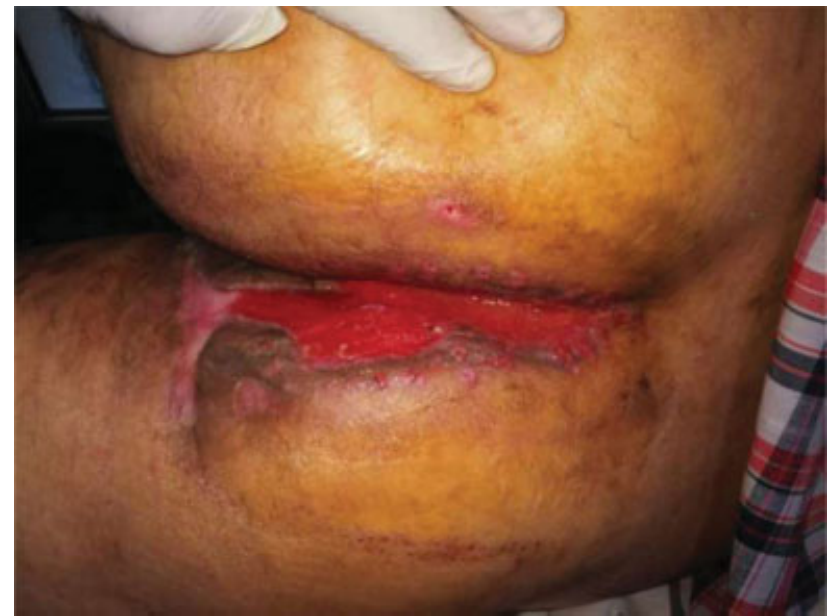

Fig. 7 Perineal wound follow-up. In total, 3 weeks post-Integra fixation and postablation of sutures. 


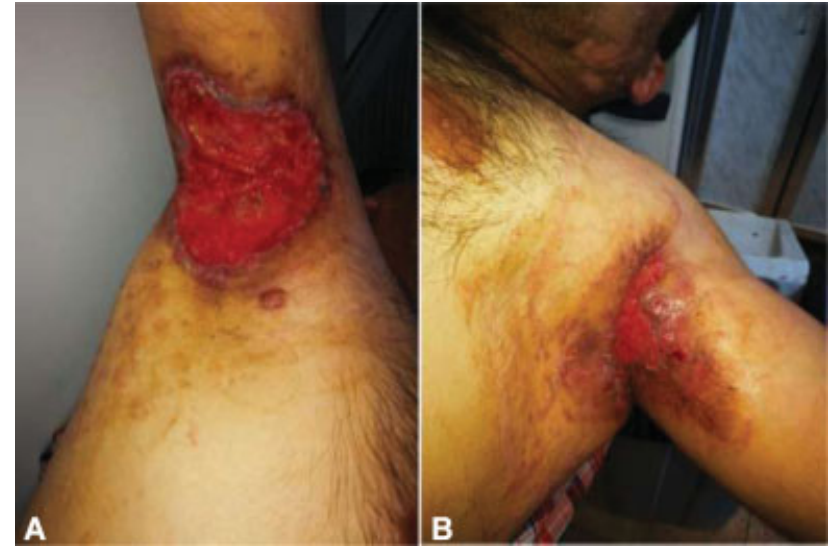

Fig. 8 Right (A) and Left (B) axillary wounds follow-up. In total, 3 weeks post-Integra fixation and postablation of sutures.

They are acquainted as tender abscesses that may burst repeatedly to purulent and odorous discharges. ${ }^{3}$ They may be demarcated as an acne-like outburst, associated with excavating blackheads ${ }^{4}$ with endmost evolution to sinus tract development and hypertrophic scare formation. ${ }^{3}$

Usually, HS are found in areas with apocrine holding skin folds such as axillae, perineum, groin, and perianal area. ${ }^{1,3}$ It generally arises postadolescence ${ }^{1}$ (between the age of 18 and 29), with threefold incidence in females. ${ }^{3}$ But its prevalence remains uncertain. ${ }^{1}$

Two classification systems were developed to direct treatment and measure its efficacy: Hurley staging and the Hidradenitis Suppurativa score successively. ${ }^{3}$ Due to the fact that HS is a labyrinthine and incongruous disease to date, it is so called Orphan, ${ }^{5}$ and a variety of surgical and medical treatments were endorsed. ${ }^{6}$ Nine international guidelines were elaborated recently and enclosing therapeutic manners, ranging from topical medication to interventional procedures. ${ }^{1}$

However, treatment steadily is founded on physician clinical expertise instead of research evidence ${ }^{3}$ since these treatment modalities are vastly imperfect, and additional studies are required to inaugurate the most effective treatment guidance. ${ }^{7}$

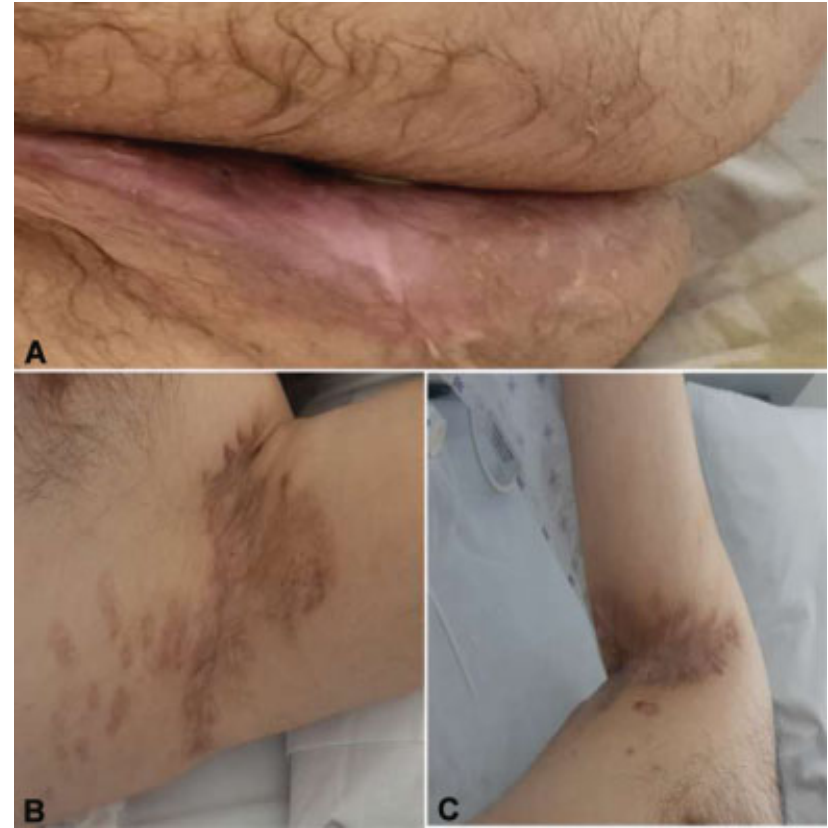

Fig. 9 1-year follow-up post-Integra removal and without recurrences: (A) perineal, (B) left axillae, and (C) right axillae.

Forasmuch as the medical treatment takes long time to function, surgery in contrast presents instant improvement, ${ }^{6}$ and it is considered the only approach with curative potential $^{3}$ mainly in the advanced cases, ${ }^{8}$ even 166 years post its first description by Verneuil. ${ }^{4}$

Surgical approach ranges from simple incision and drainage to local or wide excision. ${ }^{8}$ Thereafter, wide excision, a reconstructive technique such as skin graft or dermal substitute application over granulation tissue should be used to cover the large defect. ${ }^{8}$

Unfortunately, there is no step-by-step guide for best practice. In the absence of described surgical technique, recurrence of this entity remains a challenge. We found it necessary to begin establishing a step-wise perspective for the treatment of such a challenging entity. ${ }^{7}$ - Fig. 10 summarizes our approach.

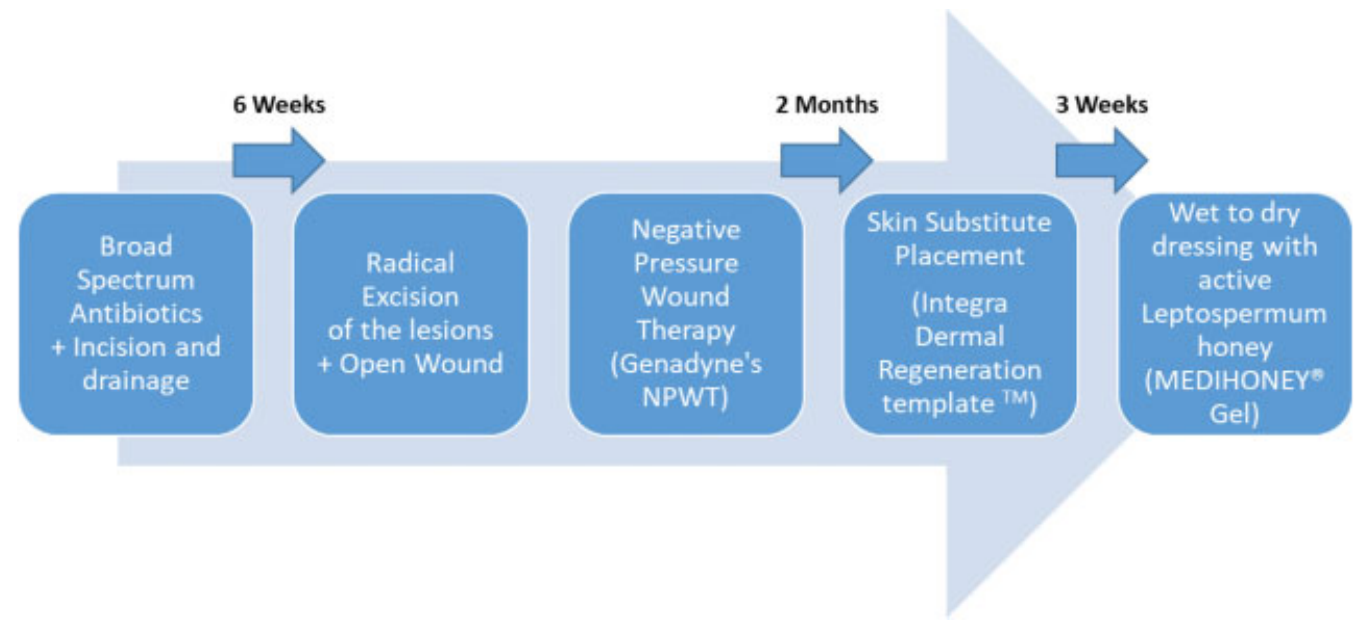

Fig. 10 Treatment recommendation based on our own experience. 


\section{Conclusion}

This report is an attempt to describe a step-wise approach to treat HS. After one recurrence free year, we find it necessary to start broader studies and enroll our technique to end up in a well-defined recommendation for an efficient surgical and reconstructive modality for patients with Hurley Stage III HS disease in multiple simultaneous locations.

\section{Note}

Written informed consent was obtained from the patient for publication of this case report and accompanying images. A copy of the written consent is available for review by the Editor-in-Chief of this journal on request.

Funding

This research received funding from Medispharm, the local agent and supplier of Genadyne's NPWT, Integra Dermal Regeneration template TM and MEDIHONEY Gel. A.K., H.A., G.R.A, A.N., G.B., E.A.F., G.R.N., H.B.M., H.A.K.M., S.I., and E.E.H. reports grants from Medispharm, during the conduct of the study.

\section{Conflict of Interest}

None declared.

\section{Acknowledgments}

The authors would like to thank the nurses of our institute for taking a good care of wounds dressing. In addition, they would like to thank Medispharm's management and employees, especially Mr. Elie Lebbos and Mme Rasha Jaafar.

\section{References}

1 Hendricks AJ, Hsiao JL, Lowes MA, Shi VY. A Comparison of International Management Guidelines for Hidradenitis Suppurativa. Dermatology 2019:1-16

2 Sivanand A, Alhusayen R, Piguet V, Alavi A. "Hidradenitis suppurativa" is a historical term that does not reflect the current understanding of disease pathogenesis. J Cutan Med Surg 2020; 24(06):644-645

3 Duran C, Baumeister A. Recognition, diagnosis, and treatment of hidradenitis suppurativa. JAAPA 2019;32(10):36-42

4 Rowland Payne PCME. Hidradenitis suppurativa treatment medical or surgical? J Eur Acad Dermatol Venereol 2019;33 (06):981-982

5 Gulliver W, Landells IDR, Morgan D, Pirzada S. Hidradenitis suppurativa: a novel model of care and an integrative strategy to adopt an orphan disease. J Cutan Med Surg 2018;22(01): 71-77

6 Melendez Gonzalez MDM, Sayed CJ. Surgery is an essential aspect of managing patients with hidradenitis suppurativa. J Am Acad Dermatol 2020;83(03):979-980

7 Tchero H, Herlin C, Bekara F, Fluieraru S, Teot L. Hidradenitis suppurativa: a systematic review and meta-analysis of therapeutic interventions. Indian J Dermatol Venereol Leprol 2019;85(03): 248-257

8 Manfredini M, Garbarino F, Bigi L, Pellacani G, Magnoni C. Hidradenitis suppurativa: surgical and postsurgical management. Skin Appendage Disord 2020;6(04):195-201 